\title{
Efeitos das terapias na menopausa: uma revisão narrativa da literatura
}

\section{Effects of menopause therapies: a narrative literature review}

\author{
Jucelia Manica $^{1}$ (D) , Emyr Hiago Bellaver ${ }^{1}$ (D), Vilmair Zancanaro ${ }^{2,3}$ (D)
}

1. Discente do Curso de Farmácia pela Universidade Alto Vale do Rio do Peixe (UNIARP), Caçador, SC, Brasil. 2. Docente do curso de Farmácia pela Universidade Alto Vale do Rio do Peixe (UNIARP), Caçador, SC, Brasil. 3. Mestranda em Ciência e Biotecnologia pela Universidade do Oeste de Santa Catarina (UNOESC), Joaçaba, SC, Brasil.

\section{Resumo}

Introdução: A menopausa é um evento que determina o final do período fértil da mulher, com efeitos, sobretudo, no bem-estar e que em alguns casos pode ser necessário a intervenção de terapias medicamentosas ou medidas alternativas. Objetivo: Consiste em uma revisão de informações relativas ao tratamento dos sintomas da menopausa, avaliando as terapias realizadas em mulheres. Método: Busca de informações em periódicos indexados nas principais bases científicas on-line, incluindo apenas estudos realizados nos anos de 2012 a 2017. Resultados: Atualmente, há terapias medicamentosas e alternativas, que têm sido eficazes para o tratamento nesta fase, sendo que, para a primeira, o Ministério da Saúde orienta que a dose administrada da terapia de reposição hormonal (TRH) deve ser a mínima eficaz para melhorar os sintomas indesejáveis, devendo ser interrompida assim que os benefícios tenham sido alcançados. Estima-se, ainda, que novas moléculas com efeitos máximos e riscos mínimos sejam descobertas e incluídas na TRH com uma avaliação dos seus reais riscos para o câncer de mama, tromboembolismo ou eventos cardiovasculares. É possível avaliar que existem vários métodos de tratamento. Conclusão: A decisão de adotar ou não uma terapia deve ser debatida entre médico e paciente individualmente, ressaltando as consequências da redução estrogênica climatérica, os efeitos colaterais e contraindicações dessas terapias, a fim de estabelecer seu custo benefício.

Palavras-chave: Menopausa. Climatério. Qualidade de vida

\begin{abstract}
Introduction: Menopause is an event that determines the end of the woman's fertile period, with effects, above all, on well-being may in some cases be necessary the intervention of drug therapies or alternative measures. Objective: Was the development of a literature review, evaluating the therapies performed in women during the menopause. Methods: The search for information in indexed periodicals in the main scientific bases on-line, including only studies carried among years 2012 at 2017. Results: There are now drug therapies and alternatives that have been effective for treatment during this period, and for the first, the Ministry of Health advises that the dose of hormone replacement therapy (HRT) administered should be the least effective for improving symptoms and should be discontinued once the benefits have been achieved. It is also estimated that new molecules with maximum effects and minimal risks are discovered and included in HRT with an assessment of their real risks for breast cancer, thromboembolism, or cardiovascular events. It is possible to evaluate that there are several treatment methods. Conclusion: The decision whether or not to adopt a therapy should be discussed between the physician and the individual patient, highlighting the consequences of estrogenic climacteric reduction, the side effects and contraindications of these therapies, in order to establish their cost-effectiveness.
\end{abstract}

Key words: Menopause. Climacteric. Quality of life.

\section{INTRODUÇÃO}

Atualmente, a expectativa de vida das mulheres em países desenvolvidos é superior a 80 anos. Usando a idade de mais ou menos 50 anos como uma proximidade para a menopausa, cerca de 25 milhões de mulheres passam por este período a cada ano. Em 2030, a população mundial de mulheres na menopausa e na pós-menopausa deverá aumentar para 1,2 bilhão, com 47 milhões de novos casos a cada ano, tornando a menopausa uma importante questão de saúde pública ${ }^{1}$.

O Ministério da Saúde estabelece o limite etário para a menopausa entre 40 e 65 anos de idade, dividido em prémenopausa que, geralmente, inicia após os 40 anos, com diminuição da fertilidade em mulheres com ciclos menstruais regulares ou com padrão menstrual similar ao ocorrido durante a vida reprodutiva; a perimenopausa que inicia dois anos antes da última menstruação e vai até um ano após (com ciclos menstruais irregulares e alterações endócrinas), a pós-menopausa que começa um ano após o último período menstrual, dando continuidade ao climatério que representa a passagem da fase reprodutiva para uma fase não reprodutiva, com possíveis alterações clínicas. É um fenômeno natural do organismo da mulher, não patológico decorrente do esgotamento dos folículos ovarianos, sendo, assim, uma diminuição progressiva da secreção dos hormônios femininos, como o estrógeno e a progesterona e, por fim, a menopausa considerada como a última menstruação da mulher e, assim, a perda da função ovariana $a^{2,3,4,5,6}$.

A carência de estrogênios na mulher climatérica determina mudanças fisiológicas marcantes. Neste período, as queixas 
frequentemente relatadas incluem as irregularidades menstruais, sintomas vasomotores (SVM), afrontamentos e suores (frequentemente noturnos), que podem surgir associados com alterações do sono (o que por si contribui para a fadiga e irritabilidade da mulher, condicionando a sua qualidade de vida), as alterações tróficas urogenitais, cognitivas e sexuais ${ }^{5,6,7}$. Diante do exposto, objetivou-se uma revisão de informações relativas ao tratamento dos sintomas da menopausa.

\section{MÉTODOS}

Com a finalidade de atingir o objetivo proposto, realizarou-se uma revisão narrativa de literatura e uma análise que permitem a busca por bibliografias indexadas em bases de dados como PubMed, Scielo, Science direct, utilizando as palavraschave: menopausa, terapias e práticas complementares, recorrendo ao uso do conector e em pesquisas separadas. Nessa modalidade de revisão, os autores buscam interpretar

Figura 1. Fluxograma utilizado para revisão de literatura

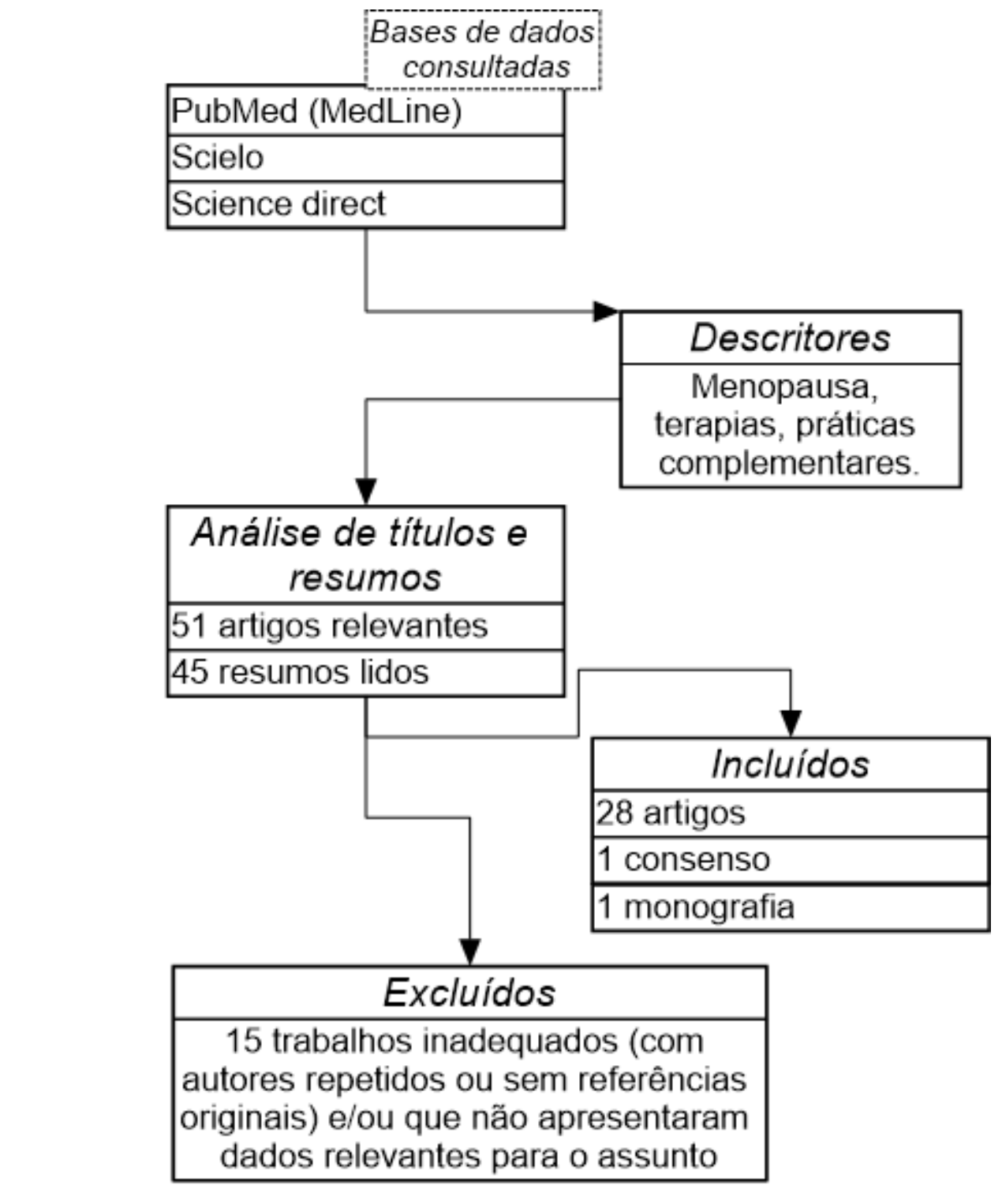

e analisar criticamente determinado fenômeno, sendo as terapias relativas ao tratamento da menopausa o foco deste estudo. A busca nas bases de dados ocorreu entre os meses de junho a novembro de 2017. Definiram-se, como critérios de inclusão, os estudos realizados nos anos de 2012 a 2017, em humanos, publicados em português, inglês e espanhol que citavam terapias para a menopausa, excluindo-se aqueles que não satisfizeram tais requisitos.

\section{RESULTADOS E DISCUSSÕES}

Ao todo 51 bibliografias foram encontradas, quarenta e cinco resumos foram lidos e, deste total, vinte e oito artigos, um consenso e uma monografia foram incluídos no presente trabalho. Artigos que não satisfizeram a temática e/os requisitos de seleção foram excluídos deste trabalho, como mostra o fluxograma abaixo (Figura 1). 


\section{O ciclo do climatério}

Doenças cardiovasculares representam a primeira causa de morte no Brasil com 32,3\%, enquanto os Estados Unidos somam índices de $42 \%$ de mortalidade em adultos relacionados às doenças coronarianas, tendo as mulheres como a maior população alvo no período da menopausa, inferindo-se maior susceptibilidade devido à perda da proteção do estrogênio. A redução desse hormônio favorece o surgimento da obesidade, caracterizando um aumento da gordura visceral, facilitando o desenvolvimento das alterações metabólicas, o aumento do ris $\neg$ co cardiovascular, dislipidemias entre outras patologias ${ }^{8}$.

Mulheres normais em idade reprodutiva produzem três classes de esteroides sexuais: os estrógenos; as progestinas e os androgênios. Esses esteroides são secretados, em sua maioria, pelo corpo lúteo. Os androgênios são precursores dos estrogênios, estando relacionados à libido e a massa muscular além do desenvolvimento normal do folículo ovariano. Mais de vinte estrógenos foram identificados, sendo os mais importantes a estrona, o estriol e o estradiol. O estradiol ou $17 \beta$-estradiol é o mais potente estrógeno, enquanto a estrona e o estriol são produtos de seu metabolismo9.

Pesquisadores da divisão de endocrinologia do Canadá analisaram diversos estudos desenvolvendo, assim, uma classificação para essa fase de vida da mulher: na fase inicial, percebem-se a regularidade no ciclo menstrual manifestando retenção hídrica, o edema nas mamas e as alterações de humor, o aumento de peso e a cefaleia, com alterações nos hormônios o FSH (Hormônio folículo estimulante) e o LH (Hormônio luteinizante) sendo possível notar a diminuição do ciclo menstrual nesta fase e os primeiros sintomas vasomotores. $\mathrm{Na}$ segunda fase, os ciclos com e sem ovulação ficam mais proeminentes seguidos da falta de ovulação e assim, ocorre o aumento do fluxo menstrual e os sintomas pré-menstruais e vasos motores se manifestam. A terceira fase é caracterizada pela irregularidade dos ciclos e os níveis do $\mathrm{LH}$ e $\mathrm{FSH}$, nos quais estão, em geral, elevados; os níveis de estradiol oscilam, os sintomas vasos motores como sensações súbitas de calor, sudorese profunda, palpitações, calafrios, tremores e ansiedade podendo surgir associados com alterações do sono ${ }^{10}$.

Na quarta fase, o fluxo menstrual aumenta de intensidade (menorragia) e a ovulação reduz para 50\%. Ocorrem aumentos nos níveis de estrogênio, progesterona, FSH e LH. Na quinta fase, ocorre a confirmação da menopausa marcada pela última menstruação devendo ser confirmada pela ausência de fluxo, por 12 meses consecutivos. Essa fase caracteriza-se pelos intensos sintomas vasomotores que têm impacto mais significativo na qualidade de vida relacionada à saúde ${ }^{10,11,12}$.

As mulheres sofrem alterações físicas e psíquicas que afetam a saúde desde a adolescência até a terceira idade. Ao longo da vida, ocorrem várias mudanças como a emocional, físicas e sociais que acabam interferindo, de modo significativo, no processo de saúde e de qualidade de vida. Os períodos de alterações no corpo da mulher são constantes e, para isso, é necessário observar os aspectos sociais, fator cultural, atividade física, inserção profissional, renda, hábitos alimentares, lazer, sexualidade e religião, todos esses fatores são importantes para a qualidade de vida saudável ${ }^{13}$.

\section{Terapia de reposição hormonal: riscos e benefícios}

A terapia de reposição hormonal (TRH) é a mais eficaz para melhorar a qualidade e os sintomas da menopausa. Essa terapia foi amplamente utilizada entre as mulheres até 2002, mas as atitudes das mulheres e dos clínicos mudaram, consideravelmente, nos últimos anos. Estudos concluíram que os efeitos nocivos da TRH excedem os benefícios e o fato ocasionou uma diminuição das prescrições médicas, levando as mulheres a buscar terapias alternativas ${ }^{14}$.

Alguns dos principais riscos da TRH são os cânceres de mama e cânceres do endométrio. Também é possível avaliar alguns benefícios, como diminuir a atrofia urogenital, melhorar o humor e reduzir os sintomas vasomotores ${ }^{15}$.

\section{Vias de Administração}

A via transdérmica mostra menor risco de tromboembolismo venoso do estradiol quando comparado com a via oral, mostrando ser a mais segura. Por outro lado, a administração, por via oral, gera um maior impacto na redução dos níveis do colesterol LDL, o que é uma vantagem na mulher com hipercolesterolemia e triglicérides normais, levando-se em conta que o último pode elevar-se com a medicação ${ }^{16,17}$.

A via de administração não oral do estradiol e da progesterona impede o metabolismo de primeira passagem pelo fígado, ocasionando, assim, menor potencial para a formação das proteínas hepáticas, fatores de coagulação e perfil metabólico, o que pode ser mais favorável em termos de riscos cardiovasculares e fenômenos tromboembólicos ${ }^{15,16,17}$.

A primeira passagem da administração vaginal de progesterona acarreta concentrações locais adequadas e boa proteção endometrial com níveis sistêmicos de progesterona menores. A combinação do uso não oral do estradiol e progesterona pode melhorar a aderência e minimizar os riscos da TRH. Entretanto, ainda são necessários estudos de boa qualidade para confirmar essa hipótese. Uso vaginal de estradiol é preferencial no tratamento isolado das queixas urogenitais como atrofia vaginal, irritação e ausência de lubrificação (Tabela 1$)^{18}$.

Um estudo realizado com 66 mulheres pós-menopausa, com o objetivo de avaliar uma formulação contendo estriol $(0,1 \%)$ + estradiol $(0,25 \%)$ e progesterona $(10 \%)$ nanoestruturada, via transdérmica, mostrou que não houve alteração da pressão arterial, sangramento vaginal e espessura aumentada do endométrio. Quando TRH é administrado por outras vias, há 
evidências de câncer de mama; e estudos recentes mostraram que a via transdérmica é a melhor maneira de tratar os sintomas da menopausa ${ }^{19}$.

Tabela 1. Vias de administração de estrógeno

\begin{tabular}{|c|c|c|}
\hline Avaliação & Via oral & Via não oral \\
\hline Vantagens & $\begin{array}{c}\text { Mais difundido } \\
\text { Custo menor } \\
\text { Menos alérgica } \\
\text { Aumento do HDL e } \\
\text { diminuição do LDL } \\
\text { colesterol }\end{array}$ & $\begin{array}{c}\text { Diminui os } \\
\text { triglicérides } \\
\text { Evita os efeitos } \\
\text { decorrentes da } \\
\text { passagem hepática }\end{array}$ \\
\hline Desvantagens & $\begin{array}{l}\text { Aumento dos } \\
\text { triglicérides } \\
\text { Diminui a } \\
\text { antitrombina III }\end{array}$ & $\begin{array}{c}\text { Custo maior } \\
\text { Impacto discreto } \\
\text { aumentando o HDL } \\
\text { e diminuindo o LDL }\end{array}$ \\
\hline
\end{tabular}

Fonte: Pardini (2014) adaptado.

Os riscos cardiovasculares associados às TRH diferem de acordo com a via utilizada. Existe forte evidência de que o estradiol transdérmico tenha um efeito cardioprotetor. 0 estudo demonstrou que o tratamento com hormônio em nano emulsão reduziu os sintomas da menopausa e restaurou significativamente os níveis séricos de estradiol e FSH. Estes resultados apoiam a investigação contínua de hormônios transdérmico nanoparticulados como um potencial agente terapêutico na terapia pós-menopausa ${ }^{19}$.

\section{Esquemas Terapêuticos}

São inúmeras as formas de administrar a TRH, visando ao alívio dos sintomas e, acima de tudo, a proteção endometrial quando associamos a progesterona ao estrógeno. Os esquemas combinados podem ser cíclicos ou contínuos. No primeiro, o estrógeno é dado de forma contínua e a progesterona de 10 a 12 dias por mês e, no segundo, ambos são administrados pela via oral de forma ininterrupta. No esquema cíclico, a mulher apresenta sangramento ao final de cada ciclo de progesterona e, no contínuo, a grande maioria entra em amenorreia e quando isso não ocorre, devem-se investigar as condições do endométrio. De qualquer forma, a escolha do esquema é sempre individualizada, priorizando-se a vontade da paciente e o tempo de menopausa ${ }^{17}$.

O Ministério da Saúde orienta que a dose administrada da TRH deve ser individualizada, mínima e eficaz para melhorar os sintomas indesejáveis, devendo ser interrompidas assim que os benefícios tenham sido alcançados (Tabela 2$)^{9}$.

No Brasil, a via oral é a forma mais utilizada para TRH, devido à facilidade de ser mais difundida, para a qual estão disponíveis combinações de hormônios nas formas farmacêuticas drágeas e comprimidos. Em segundo, a via transdérmica, devido à diminuição de efeitos adversos e de sua segurança ${ }^{20}$.

Acredita-se que a administração oral da TRH aumentaria os fatores que contribuem para os eventos tromboembólicos. A continuação da TRH, além dos 60 anos de idade, uma vez iniciada na perimenopausa, deve ser decidida como parte da análise geral da relação risco/benefício; alguns riscos como acidente vascular cerebral aumentam exponencialmente com o avanço da idade ${ }^{21}$.

Tabela 2. Classificação e apresentação dos estrógenos usados em TRH no Brasil.

\begin{tabular}{ll}
\hline Estrógeno(s) & Apresentação \\
\hline I- Orais: &
\end{tabular}

a) Derivados da estrona:

Estrógenos equinos conjugados 0,$3 ; 0,625 ; 1,25 ; 2,5 \mathrm{mg}$

b) Derivados do estradiol:

$\begin{array}{ll}\text { Valerianato de estradiol } & 1-2 \mathrm{mg} \\ \text { Estradiol micronizado } & 1-2 \mathrm{mg} \\ \text { Estriol } & 1-2 \mathrm{mg}\end{array}$

c) Sintéticos:

Etinil estradiol

0,$02 ; 0,05 ; 0,5 \mathrm{mg}$

II - Injetáveis:

Benzoato de estradiol

$0,5 \mathrm{mg} / \mathrm{mL}$

Fosfato de poliestradiol

$40 \mathrm{mg} / \mathrm{mL}$

Estrógenos equinos conjugados $25 \mathrm{mg} / \mathrm{mL}$

Valerianato de estradiol

$10 ; 20 ; 40 \mathrm{mg} / \mathrm{mL}$

III - Vaginais

Estrógenos equinos conjugados

Estriol $0,625 \mathrm{mg} /$ dose

IV - Implante

Estradiol 25; $50 ; 100 \mathrm{mg} / \mathrm{pellet}$

V-Transdérmico e percutâneo

Estradiol TTS

25; 50; 100 mcg/adesivo

Estradiol

Gel $1 \mathrm{mg} /$ dose

Fonte: Pardini (2014) Modificada.

A declaração da Sociedade da Menopausa da América do Norte (NAMS) afirma que a TRH é o tratamento mais efetivo para os sintomas da menopausa. Para maximizar a segurança, o início deve ser considerado para mulheres sintomáticas saudáveis que estão dentro de 10 anos de menopausa ou com idade inferior a 60 anos e que não têm contraindicações para uso da TRH. O uso de TRH deve ser individualizado e não descontinuado apenas com base na idade de uma mulher (Tabela 3$)^{22}$.

Segundo a Sociedade Portuguesa de Ginecologia (2016), a dose deve ser individualizada, eficaz no controle da sintomatologia e a mais baixa possível. $017 \beta$-estradiol e o princípio ativo natural 
Tabela 3. Doses de estrógeno usadas em terapia hormonal da menopausa nos Estados Unidos

\begin{tabular}{lrrrr}
\hline Estrogênios & Alta & Padrão & Baixa & Ultrabaixa \\
\hline Estradiol via oral & $2,0 \mathrm{mg}$ & $1,0 \mathrm{mg}$ & $0,5 \mathrm{mg}$ & $0,25 \mathrm{mg}$ \\
$\begin{array}{l}\text { Estrógenos Equinos } \\
\text { Conjugados via oral }\end{array}$ & $1,25 \mathrm{mg}$ & $0,625 \mathrm{mg}$ & $0,3-0,45 \mathrm{mg}$ & - \\
$\begin{array}{l}\text { Estradiol } \\
\text { Via transdérmica }\end{array}$ & $0,10 \mathrm{mg}$ & $0,05 \mathrm{mg}$ & $0,025 \mathrm{mg}$ & $0,014 \mathrm{mg}$ \\
\hline
\end{tabular}

Fonte: NAMS (2015b) adaptado.

são convertidos em estrona e depois em sulfato de estrona no fígado ou em outros tecidos. A administração por via oral necessita ser micronizada para sua melhor absorção. Nessa via ocorre um acúmulo de sulfato de estrona de liberação lenta o que permite sua administração em dose única. Pode ainda ser utilizada por via transdérmica, percutânea, intranasal (não comercializado em Portugal) e vaginal. O valerato de estradiol atua como um pró-fármaco do estrogênio natural. Sofre uma rápida clivagem em $17 \beta$-estradiol e ácido valérico no tubo digestivo e durante a metabolização em nível hepático.
Estrógenos equinos conjugados (EEC) podem ser administrados por via oral e vaginal. Não são comercializados, em Portugal, de forma isolada. O estriol é apenas utilizado na forma tópica. $\mathrm{O}$ etinilestradiolé um derivado sintético do $17 \beta$-estradiol que integra a maioria das formulações dos contraceptivos combinados. A sua associação em baixa dose e com a noretisterona foi testada na TRH, com eficácia na redução dos sintomas vasomotores, atrofia endometrial, no entanto aumentou o índice de massa corporal (IMC) e não apresentou uma taxa de efeitos adversos semelhante ao placebo (Tabela 4$)^{22}$.

Tabela 4. Doses e equivalência dos estrogênios em Portugal.

\begin{tabular}{llll}
\hline Estrogênios & Padrão & Baixa & Ultrabaixa \\
\hline $17 \beta$-estradiol oral & $2 \mathrm{mg}$ & $1 \mathrm{mg}$ & $0,5 \mathrm{mg}$ \\
$17 \beta$-estradiol transdérmico & $0,05 \mathrm{mg}(50 \mu \mathrm{g})$ & $0,025 \mathrm{mg}(25 \mu \mathrm{g})$ & $0,014 \mathrm{mg}(14 \mu \mathrm{g})$ \\
Valerato estradiol oral & $2 \mathrm{mg}$ & $1 \mathrm{mg}$ & - \\
Estrógenos equinos conjugados & $0,625 \mathrm{mg}$ & $0,45 \mathrm{mg}$ & - \\
Etinilestradiol & $15 \mu \mathrm{g}$ & - & - \\
\hline
\end{tabular}

Fonte: Sociedade Portuguesa de Ginecologia (2016) adaptada.

Analisando as dosagens de hormônios via oral das tabelas 2, 3 e 4 infere-se que, em Portugal, existe uma dosagem ultrabaixa de estradiol 0,015 mg e a segunda menor dosagem acontece nos Estados Unidos com 0,25 mg. Na via transdérmica, as dosagens são iguais nesses países.

A duração da TRH é um dos maiores desafios do tema analisado e os dados atuais são inconsistentes para definir quando interromper a hormonioterapia. Para a reposição com estrógeno isolado, existe maior flexibilidade quanto ao tempo de uso, sendo que as normativas são de manter a reposição individualizada com base nos sintomas, monitorizada e mantida enquanto os benefícios forem superiores aos riscos sempre sob supervisão médica. Espera-se, em um futuro próximo, novas moléculas com efeitos máximos e riscos mínimos sejam descobertas e incluídas na TRH. A genotipagem individual poderá identificar a paciente que possui risco real para câncer de mama, tromboembolismo ou evento cardiovascular ${ }^{18,23}$.

A TRH continua sendo a terapêutica de escolha para os sintomas da menopausa e sua segurança depende da boa indicação, monitorização e individualização. Mulheres acima dos 60 anos de idade não devem iniciar a $\mathrm{TRH}^{16}$. Os consensos atuais são unânimes quando relatam que os benefícios da TRH são máximos quando iniciada na perimenopausa. A mulher de 50 a 59 anos ou com menos de 10 anos de menopausa é o grupo alvo e representa a faixa de mulheres na qual os benefícios, na maioria das vezes, superam os riscos ${ }^{5,18}$.

\section{TERAPIAS ALTERNATIVAS}

\section{Tibolona}

A Tibolona é um esteroide sintético aprovado em 90 países para tratar os sintomas da menopausa, incluindo Brasil, Europa e Austrália, mas não nos Estados Unidos e, em 45 países, está aprovado para prevenção de osteoporose. No Brasil, o uso de tibolona é em dosagem de 1,25 mg a 2,5 mg na forma comercial conhecida como Libiam $^{\circledR}$. A tibolona alivia os sintomas vasomotores, melhora a atrofia urogenital, previne a perda de massa óssea e acarreta aumento da densidade óssea. Devido a seu perfil androgênico melhora a libido ${ }^{18,20}$.

\section{Raloxifeno}

Visando às alternativas para ação agonista do estrógeno, a 
indústria farmacêutica desenvolveu os moduladores seletivos do receptor de estrógeno (SERMs). Esses fármacos constituem classe de moléculas não hormonais com alta afinidade de ligação aos receptores de estrógeno. Como resultado, na dose de $60 \mathrm{mg} / \mathrm{dia}$, melhora a densidade mineral óssea e reduz a incidência de fratura vertebral, mas não reduz a não vertebral. A semelhança do estrógeno aumenta o risco de acidente vascular cerebral e de tromboembolismo. Como resultado de suas ações antiestrogênicas, o raloxifeno reduz a incidência de câncer de mama e endométrio, entretanto piora os sintomas vasomotores $^{18,24}$.

\section{Isoflavona}

Os fitoestrogênios são compostos derivados de plantas que possuem efeitos estrogênicos ${ }^{25}$. As Isoflavonas são encontradas em grandes concentrações na soja e produtos derivados da soja. A eficácia terapêutica é variável de acordo com a extração, fabricação, composição, dosagem, estandardização e absorção. As Isoflavonas têm grande afinidade para os receptores de estrogênio ${ }^{26}$. No Brasil, a Anvisa aprova o uso da Isoflavona somente para o tratamento dos sintomas vasomotores ${ }^{5,9}$.

As isoflavonas são menos potentes do que o estrogênio endógeno. Seu uso terapêutico está relacionado com diminuição nos sintomas da menopausa, uma forma alternativa de terapia de reposição natural para alívio dos sintomas, eleva os níveis de colesterol bom (HDL) e diminui os níveis de colesterol ruim (LDL), reduz também a probabilidade de osteoporose e também ajuda na redução de risco de determinados tipos de câncer. Suas propriedades farmacológicas implicam tecidos alvos como hipófise anterior e hipotálamo, auxiliando em seus efeitos e sintomas do climatério ${ }^{13,17}$.

Estudos recentes (NAMS, 2015b) não encontraram evidências eficazes da Isoflavona na redução de sintomas vasomotores quando comparados com o placebo. Após duas décadas de estudo sobre as isoflavonas, seus efeitos terapêuticos ainda não são claros. Os estudos recentes de 2015 e 2016 mais uma vez não são conclusivos. Uma metanálise de 2015 mostrou que os fitoestrogênios não demonstraram qualquer benefício, mas, quando avaliado isoladamente o parâmetro SVM, verificou-se redução da frequência dos calores e afrontamentos quando comparado com o placebo ${ }^{27}$. Existem limitações aos estudos como a quantidade, tipo de Isoflavonas disponíveis na fabricação, duração e doses dos tratamentos instituídos, o que impossibilita a comparação dos diferentes fitoestrogênios ${ }^{22}$.

\section{Resveratrol}

Foi caracterizado como um fitoestrogênio com base na sua capacidade de se ligar aos receptores de estrogênio para melhorar a função endotelial, aumentando a biodisponibilidade do óxido nítrico. O resveratrol pode estimular a proliferação e a diferenciação de células ósseas e inibe a apoptose de condrócitos por meio de antiinflamatórios ${ }^{28}$. Até então nenhum estudo explorou o potencial do resveratrol para gerenciar condições como dor, sintomas vasomotores, e desempenho cognitivo associado à menopausa. Os efeitos de um teste de suplementação de resveratrol de 14 semanas em desempenho cognitivo, função cerebrovascular e o humor em mulheres pósmenopáusicas confirmou a eficácia do resveratrol para reduzir a dor como osteoartrite relacionada à idade em mulheres na pós-menopausa ${ }^{28}$.

\section{Pycnogenol}

O pycnogenol é um grupo de substâncias encontrada na casca do pinheiro marítimo francês (Pinus pinaster), exerce um amplo espectro de efeitos antioxidantes e antinflamatórios. Além disso, mostrou uma série de benefícios cardiovasculares e um efeito antidiabético. Os estudos clínicos mostram que o pycnogenol melhorou, significativamente, os sintomas da menopausa em mulheres de três nacionalidades diferentes ${ }^{29}$. Com o objetivo de avaliar a eficácia do Pycnogenol ${ }^{\circledR}$, um estudo foi realizado quando se administrou via oral, $100 \mathrm{mg} / \mathrm{dia}$, em um grupo de 35 mulheres e observou-se melhora na qualidade de vida das mulheres perimenopáusicas, normalizando uma série de fatores de risco cardiovasculares como homocisteína e proteina $\mathrm{C}$ reativa ${ }^{30}$.

\section{CONSIDERAÇÕES FINAIS}

É necessário um consenso a respeito da TRH, baseado em uma reavaliação quanto aos seus reais riscos e benefícios; assim, então, será possível garantir amplos benefícios terapêuticos na prevenção e no tratamento dos sintomas do climatério e pós-menopausa, proporcionando às mulheres uma melhor qualidade de vida e um tratamento seguro, eficaz e com o mínimo de efeitos adversos. Estudos científicos têm demonstrado a eficácia das terapias alternativas no tratamento de muitos sintomas da menopausa, como a diminuição dos sintomas vasomotores e urogenitais.

O presente estudo possibilitou observar que a menopausa é uma fase normal da vida de cada mulher, uma vez que existem vários meios de terapias. A decisão de adotar, ou não, uma terapia deve ser tomada entre o médico e a paciente individualmente, ressaltando as consequências da redução estrogênica climatérica, os efeitos colaterais e as contraindicações dessas terapias, a fim de estabelecer seu custo e benefício.

\section{REFERÊNCIAS}

1. Schneider HPG, Birkhäuser M. Quality of life in climacteric women. Climacteric. 2017 Jun; 20(3): 187-194. doi: 10.1080/13697137.2017.1279599. PubMed PMID: 28118068.

2. Nogueira Valença C, Medeiros Germano R. Concepções de mulheres sobre menopausa e climatério. Revista da Rede de Enfermagem do Nordeste. 2010;
11(1). Disponível em:<http://www.redalyc.org/articulo.oa?id=324027969021>. Acesso em: 06 nov. 2018. ISSN 1517-3852.

3. Godinho A, Rato I, Calhaz-Jorge C. Endocrinologia da mulher. In Neves J. Medicina da mulher na pós-menopausa. Lisboa: LIDEL; 2012. p.768-2,. 
4. Lamas MC, Paúl C, Montenegro N. 2014. Saúde Mental e bem-estar da mulher na perimenopausa. Uma Revisão Sistemática. E-book do V Congresso Internacional da Sociedade Portuguesa de Enfermagem de Saúde Mental "Consensos em Saúde Mental", p.201-214. Disponível em: http://issuu.com/ spesm/docs/2015_consensos_sa_de_mental_ebook_> Acesso em: 06 nov. 2018.

5. Lente CL, Velasques LFL. Efeitos da terapia hormonal na menopausa: Revisão de literatura. Biosaúde. 2015; 17(2):74-81.

6. Correia AC, Lamas MC, Oliveira RF. Avaliação da terapêutica hormonal de substituição e das isoflavonas de soja na peri e pós-menopausa. Actas de Gerontologia. 2016; 2(1):2.

7. Graef AM, Locatelli C, Santos P. Utilização de fitoestrógenos da soja (glycinemax) e angelicasinensis (dongquai) como uma alternativa terapêutica para o tratamento dos sintomas do climatério. Evidência. 2012 Jan-Jun; 12(1):83-96

8. Guerra TRB, Boaventura GT. Consumo de fitoestrógeno linhaça e níveis lipídicos na menopausa: o que há de evidência. Rev. Aten. Saúde. 2016 Jul-Set; 14(49):92-97.

9. Oliveira J, Peruch MH, Gonçalves S, Haas P. Padrão hormonal feminino: menopausa e terapia de reposição. RBAC. 2016; 48(3):198-210.

10. Silva DR, Cunha MSF. Ouso da isoflavona no tratamento do climatério. [Monografia]. Pindamonhangaba(SP): Faculdade de Pindamonhangaba; 2015.

11. Freeman EW, Sammel MD, Sanders RJ. Risk of long-term hot flashes after natural menopause: evidence from the Penn Ovarian Aging Study cohort. Menopause. 2014 Sep; 21(9) :924-32. doi: 10.1097/GME.0000000000000196.

12. Avis NE, Crawford SL, Greendale G, Bromberger JT, Everson-Rose SA, Gold $E B$, et al. Duration of menopausal vasomotor symptoms over the menopause transition. JAMA Intern Med. 2015 Apr; 175(4): 531-9. doi: 10.1001/ jamainternmed.2014.8063. PubMed PMID: 25686030.

13. Ferreira R, Pereira A, Alves V, Santos M, Rodrigues D, Marchiori G. Saúde de mulheres no climatério no sistema prisional. Cogitare enferm [Internet]. 2017;22(1):1-8. Disponível em: <https://revistas.ufpr.br/cogitare/article/ view/48235> Acesso em: 06 nov. 2018. doi: 10.5380/ce.v22i1.48235.

14. Pinkerton JV, Constantine GD. Compounded non-FDA-approved menopausal hormone therapy prescriptions have increased: results of a pharmacy survey. Menopause. 2016 Apr; 23(4):359. doi: 10.1097/GME.0000000000000567.

15. Sociedade Portuguesa de Ginecologia. Consenso nacional sobre menopausa Coimbra: SPG; 2016. Disponível em: http://www.spginecologia.pt/consensos/ consenso-nacional-sobre-menopausa-2016.html>. Acesso em: 7 out. 2017.

16. Yeganeh L, Boyle J, Teede H, Vincent A. Knowledge and attitudes of health professionals regarding menopausal hormone therapies. Climacteric. 2017 Aug; 20(4): 348-355. doi: 10.1080/13697137.2017.1304906. PubMed PMID: 28379046 .

17. Liu J, Yuan F, Gao J, Shan B, Ren Y, Wang H, et al. Oral isoflavone supplementation on endometrial thickness: a meta-analysis of randomized placebo-controlled trials. Oncotarget. 2016;7(14):17369-79. Disponível em: <https://www.ncbi.nlm.nih.gov/pm c/articles/PMC4951218/> Acesso em: 06 nov. 2017. doi: 10.18632/oncotarget.7959. PubMed PMID: 26967050.

18. Pardini D. Terapia de reposição hormonal na menopausa. Arq Bras Endocrinol Metab. 2014; 58(2): 172-181.

19. Botelho MA, Queiroz DB, Barros G, Guerreiro S, Fechine P, Umbelino S, et al. Nanostructured transdermal hormone replacement therapy for relieving menopausal symptoms: a confocal Raman spectroscopy study. Clinics (São Paulo). 2014;69(2):75-82. Disponível em: <http://www.scielo.br/scielo. php?script=sci_arttext\&pid=S1807-593220 14000200075\&lng=en\&nrm=iso $>$. Acesso em: 06 nov. 2017. ISSN 1807-5932. doi: 10.6061/clinics/2014(02)01.

20. Gelatti GT, Berlezi EM, Colet CF, Oliveira KR, Horn RC. Via de administração da reposição hormonal utilizada por mulheres pós-menopausa e a sua relação com as doenças apresentadas. BIOMOTRIZ. 2015; 9(1): 138-149.

21. Borges TFC, Silva APT, Ferreira MSC. Terapia com Hormônios Sexuais Femininos e Fenômenos Tromboembólicos: uma Revisão de Literatura/Female Sex Hormone Therapy and Thromboembolic Phenomena: Literature Review. Rev Cienc Saúde. 2015; 5(2):158-168.

22. North American Menopause Society. Nonhormonal management of menopause-associated vasomotor symptoms. Menopause. 2015; 22(11):11551174.

23. Lobo RA. Where are we 10 years after the Women's Health Initiative? Clin Endocrinol Metab. 2013 May; 98(5): 1771-80. doi: 10.1210/jc.2012-4070. PubMed PMID: 23493433.

24. Condi FL, Soares JM Júnior, Teodoro WR, Velosa AP, Parra ER, Jesus SM, et al. The effects of conjugated estrogen, raloxifene and soy extract on collagen in rat bones. Climacteric. 2012; 15(5):441-448.

25. Lethaby A, Marjoribanks J, Kronenberg F, Roberts H, Eden J, Brown J. Phytoestrogens for menopausal vasomotor symptoms. Cochrane Data base of Syst Rev. 2013 Dec; 12(12). doi: 10.1002/14651858.CD001395.pub4.

26. Von Studnitz FS, Eulenburg C, Mueck AO, Buhling KJ. The value of complementary and alternative medicine in the treatment of climacteric symptoms: Results of a survey among German gynecologists. Complementary Ther Med. 2013 Oct; 21(5): 492-495. doi: 10.1016/j.ctim.2013.07.005.

27. Chen MN, Lin CC, Liu CF. Efficacy of phytoestrogens for menopausal symptoms: a meta-analysis and systematic review. Climacteric. 2015 Apr; 18(2):260-9. doi: 10.3109/13697137.2014.966241.

28. Wong RHX, Evans HM, Howe PRC. Resveratrol supplementation reduces pain experience by postmenopausal women. Menopause. 2017 Aug; 24(8): 916-922. doi: 10.1097/GME.0000000000000861.

29. Kohama T, Negami M. Effect of low-dose French maritime pine bark extract on climacteric syndrome in 170 perimenopausal women. J Reprod Med. 2013 Jan-Feb; 58(1-2): 39-46.

30. Luzzi R, Belcaro G, Hosoi M, Feragalli B, Cornelli U, Dugall $M$, et al. Normalization of cardiovascular risk factors in peri-menopausal women with Pycnogenol ${ }^{\circledR}$. Minerva Ginecol. 2017 Feb; 69(1): 29-34. doi: 10.23736/S00264784.16.03913-7

\section{Como citar este artigo/How to cite this article:}

Manica J, Bellaver EH, Zancanaro V. Efeitos das terapias na menopausa: uma revisão narrativa da literatura, São Paulo, Brasil. J Health Biol Sci. 2018 JanMar; 7(1):82-88. 